\title{
Rating Rectangular Farm Delivery Meter Gates for Flow Measurement
}

\author{
Daniel J. Howes, M.ASCE${ }^{1}$; and Charles M. Burt, M.ASCE ${ }^{2}$
}

\begin{abstract}
Traditional meter gates for farm delivery flow measurement from an open channel conveyance have traditionally incorporated round canal gates (Armco type) for control. In recent years, some irrigation water agencies (i.e., irrigation districts) have replaced deteriorating round gates with lower-cost rectangular gates that cover round holes. Similar to the situation described in a companion paper, where round gates were examined, there have been no investigations into flow measurement uncertainty using the existing rating tables for these gates. In this study, two commonly used rectangular gate sizes, $0.46 \mathrm{~m}$ (18-in.) and $0.61 \mathrm{~m}$ (24-in.), were tested under scenarios of various gate openings, upstream heads, and head differences. Coefficient of discharge $\left(C_{d}\right)$ values were computed based on actual gate open areas. These improved $C_{d}$ values were used to generate new discharge rating tables for $0.46 \mathrm{~m}$ (18-in.) and $0.61 \mathrm{~m}$ (24-in.) rectangular meter gates. Limitations for these rectangular gates are discussed. If guidelines presented in this paper and in the companion paper are followed, the average instantaneous flow measurement uncertainty that could be expected is better than $\pm 5 \%$. However, uncertainty is higher (up to approximately $9.5 \%$ ) at the lower end of the recommended gate openings $[0.10 \mathrm{~m}(4 \mathrm{in}$.) $]$ for these rectangular gates.
\end{abstract}

\section{Introduction}

Flow measurement of water delivered to farms is important for water management both on-farm and within the water agency/ district conveying the water. Water is often measured by volume or flow rate at a turnout structure, which is a device that moves water from the main conveyance either to a lateral or on-farm conveyance. There are many common devices used to measure flow rate through farm turnout structures, which vary widely in cost (in terms of both fixed costs and maintenance), accuracy, and strengths/limitations. For example, propeller meters are commonly used in districts that have clean water and turnout configurations that fit the specific installation requirements of propeller meters. The fixed cost is generally reasonable, but these devices must be removed and recalibrated every several years, which increases the maintenance costs. Most districts must deal with moss, algae, and aquatic weeds, which plug up the meters and render them ineffective. The installation of necessary debris screens upstream of the devices also significantly increases the initial cost of these devices.

In this paper, as well as a companion paper, a device termed a meter gate was evaluated. Meter gates have been installed throughout much of California and can be found in regions all over the world. The traditional meter gate consists of a round gate on the upstream end of a round culvert pipe with a tap hole for a stilling

\footnotetext{
${ }^{1}$ Assistant Professor, Dept. of BioResource and Agricultural Engineering, Irrigation Training and Research Center, California Polytechnic State Univ., San Luis Obispo, CA 93407 (corresponding author). E-mail: djhowes@calpoly.edu

${ }^{2}$ Chairman, Irrigation Training and Research Center, California Polytechnic State Univ., San Luis Obispo, CA 93407. E-mail: cburt@ calpoly.edu
}

well approximately $0.305 \mathrm{~m}$ (12 in.) downstream from the back face of the gate to measure the downstream head. The difference in head between the water levels upstream of the gate and in the downstream stilling well, in combination with the net vertical gate opening, is used with a rating table to determine the flow rate. In a companion paper, the round gate configuration (termed an Armcotype meter gate) was examined. However, irrigation districts have been replacing the round gate with square/rectangular manufactured gates because of the lower cost.

Formal testing to develop flow rate rating tables for square-flatleaf gates was conducted for $0.46 \mathrm{~m}$ (18-in.) and $0.61 \mathrm{~m}$ (24-in.) gates by the U.S. Bureau of Reclamation (USBR) in the 1950s and published in Ball (1961). This study found consistent coefficient of discharge values (based on an area computed based on pipe diameter rather than on actual gate opening area) for both gate sizes if the downstream tap hole was placed at one-third of the full pipe diameter $\left(D_{p} / 3\right)$, downstream from the face of the gate. The chart of full pipe coefficient of discharge $\left(C_{d}\right)$ values related to net gate opening percent for the square gates with a measuring tap well at $D_{p} / 3$ can be found in the USBR Water Measurement Manual (Chapter 9. Section 14. Meter Gates) (USBR 1997).

As with the Armco-type (round) gates discussed in the companion paper, the USBR testing of these rectangular gates was conducted with supply flow entering parallel to the turnout pipe (straight into the pipe). This differs from most installations in distribution canals where the supply channel flow is perpendicular to the turnout pipe. Since the 1950s when the USBR work was completed, no independent evaluations of the results for rectangular meter gates has been conducted. In addition, the USBR Flow Measurement Manual states that the methodology presented in the meter gate section can result in uncertainty of computed flow within $\pm 2.5 \%$ (USBR 1997). However, given the coarse resolution for full pipe $C_{d}$ in both USBR (1997) and Ball (1961), this level of accuracy is most likely unattainable.

One goal of this study was to assess the uncertainty related to using these gates for flow measurement. Another was to investigate if improved discharge equations could be developed. Finally, either 
with an improved discharge equation or improved coefficient of discharge $\left(C_{d}\right)$ values, a goal was to develop gate discharge tables for use with these rectangular meter gates.

\section{Procedures}

Procedures applicable to the testing of rectangular gates, which differ from those of the Armco-type gates, will be discussed in this section. For measurement procedures that were the same under both gate types, readers should refer to the companion paper, which describes these in detail. There may be overlap in some areas for general information. The standard discharge equation for a submerged orifice is

$$
Q=C_{d} A_{o} \sqrt{2 g \Delta H}
$$

where $Q=$ flow rate [cubic meters per second (CMS)]; $C_{d}=$ coefficient of discharge; $A_{o}=$ net gate opening area $\left(\mathrm{m}^{2}\right) ; g=$ gravitational acceleration $\left(9.81 \mathrm{~m} / \mathrm{s}^{2}\right)$; and $\Delta H=$ head difference across the gate $(\mathrm{m})$. The coefficient of velocity $\left(C_{v}\right)$ has been neglected since the velocity of approach is close to zero because these gates are typically installed perpendicular to the supply channel velocity streamlines.

If the flow rate is measured independently, the $C_{d}$ value can be computed from Eq. (2) as

$$
C_{d}=\frac{Q}{A_{o} \sqrt{2 g \Delta H}}
$$

A new meter gate testing facility was constructed at the Cal Poly Irrigation Training and Research Center (ITRC) and is described in the companion paper.

Three calibrated magnetic (mag) meters were used to measure $Q$ downstream of the meter gate. In order to test a variety of flow rates, three different mag meter sizes were used: $0.25 \mathrm{~m}$ (10-in.), $0.46 \mathrm{~m}$ (18-in.), and $0.61 \mathrm{~m}$ (24-in.). The calibration and layout of these mag meters is discussed in the companion paper.

The head difference $(\Delta H)$ is taken as the difference in the head upstream of the gate and downstream of the gate. The downstream head measurement was taken at various locations, but this paper will focus primarily on the standard pressure tap location $0.305 \mathrm{~m}$ (12 in.) downstream of the back face of the gate. Potential errors related to using pressure tap locations at the $0.15 \mathrm{~m}$ (6-in.) and $0.20 \mathrm{~m}$ (8-in.) locations will be discussed in the "Results" section. A more detailed evaluation of the influences due to pressure tap location will be presented in future work. The measurement procedure and setup is discussed in detail in the companion paper.

\section{Net Gate Opening Area $\left(A_{o}\right)$}

In this study, the actual gate opening area $\left(A_{o}\right)$ was used to compute the $C_{d}$ from Eq. (2). The actual gate opening area is smaller than the full pipe area $\left(A_{p}\right)$ for all gate openings less than fully open.

The following is the relationship between net gate open area $\left(A_{o}\right)$, pipe radius $\left(R_{p}\right)$, and net gate opening $(y)$ from Skogerboe and Merkley (1996):

$A_{o}=\frac{R_{p}^{2}}{2} \times\left\{2 \times \cos ^{-1}\left(1-\frac{2 \times y}{R_{p}}\right)-\sin \left[2 \times \cos ^{-1}\left(1-\frac{2 \times y}{R_{p}}\right)\right]\right\}$

where $y=$ net gate opening; and $R_{p}=$ pipe inside radius shown in Fig. 1. Since the ratings are based on net gate opening (also referred to as the stem height), correct measurement is critical. The correct

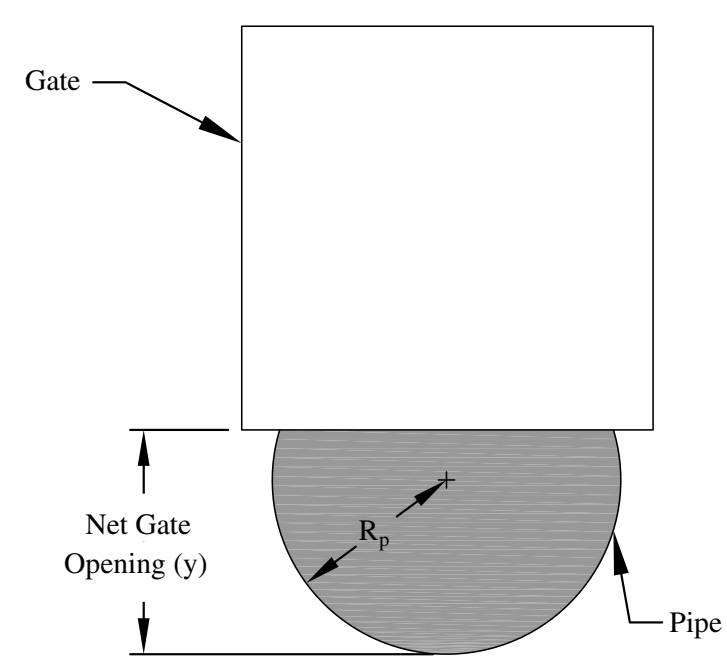

Fig. 1. Rectangular meter gate dimensions needed for correct opening area calculation; the gray region is the net gate opening area $\left(A_{o}\right)$

procedure for this measurement is from the bottom of the inside diameter of the pipe to the bottom of the gate. The gate stem (above the frame) must be marked to indicate the correct zero opening, which occurs just as the gate breaches the bottom of the pipe, while the gate is being opened (not closed). The distinction between measurement during the action of opening or closing the gate is necessary because the stem-gate connection almost always has free movement.

\section{Meter Gate Testing Scenarios}

A variety of conditions were tested for each gate to examine their effects on $C_{d}$ and their relationship to discharge. Upstream water level in the supply channel, water level in the downstream sump, gate opening, supply channel velocity, and gate size were variables investigated during the testing. The results presented here will not include effects of supply channel velocity and will focus on $\Delta H$ taken at the $0.305 \mathrm{~m}$ (12-in.) head measurement location.

Two commonly used rectangular canal gate sizes $[0.46 \mathrm{~m}$ (18-in.) and $0.61 \mathrm{~m}$ (24-in.)] were examined under various conditions. These rectangular gates were manufactured by Mechanical Associates (Visalia, California) and provided by San Luis Canal Company (Dos Palos, California) for the testing.

Table 1 shows the different tests and testing ranges conducted as part of the rectangular meter gate evaluation under low supply channel velocity. Since the gates were set perpendicular to the supply channel flow direction, tests were conducted under various supply channel flow velocities to determine if there was any influence. The lowest supply channel velocity occurred when $0.02-0.06 \mathrm{~cm}$ remained in the supply channel after flow was diverted into the meter gate. Only results from these testing scenarios will be shown in this paper. For each scenario in Table 1, a range of values for each variable was targeted. The results will be presented for the actual measured variable.

The upstream head $\left(H_{1}\right.$, measured from the top of the pipe to the water surface) varied from approximately 0.4 times the turnout pipe diameter to 2 times the pipe diameter (except for the $0.61 \mathrm{~m}$ gate). For the $0.61 \mathrm{~m}$ gate, the Very High Upstream Head was the maximum upstream water level that could be safely passed through the flume without overtopping.

Net gate openings were measured in $0.05 \mathrm{~m}$ increments from $0.05 \mathrm{~m}$ to fully open. The net gate openings were measured using 
Table 1. Tests Conducted under Low Supply Channel Velocity for the Rectangular Meter Gate Testing

\begin{tabular}{|c|c|c|c|c|c|}
\hline Gate type & Nominal gate size $(\mathrm{m})$ & Relative upstream head & Relative head loss & Upstream head $\left(H_{1}\right)$ range $(\mathrm{m})$ & $\Delta H$ range $(\mathrm{m})$ \\
\hline Rectangular & 0.46 & Very low & Small & $0.246-0.232$ & $0.059-0.027$ \\
\hline Rectangular & 0.46 & Low & Small & $0.416-0.322$ & $0.151-0.062$ \\
\hline Rectangular & 0.46 & Standard & Small & $0.73-0.457$ & $0.191-0.143$ \\
\hline Rectangular & 0.46 & Standard & Large & $0.73-0.457$ & $0.262-0.19$ \\
\hline Rectangular & 0.46 & High & Small & $0.66-0.584$ & $0.319-0.184$ \\
\hline Rectangular & 0.46 & High & Large & $0.66-0.584$ & $0.353-0.266$ \\
\hline Rectangular & 0.46 & Very high & Small & $0.819-0.775$ & $0.323-0.22$ \\
\hline Rectangular & 0.46 & Very high & Large & $0.819-0.775$ & $0.573-0.305$ \\
\hline Rectangular & 0.61 & Very low & Small & $0.449-0.249$ & $0.054-0.038$ \\
\hline Rectangular & 0.61 & Very low & Medium & $0.449-0.249$ & $0.263-0.151$ \\
\hline Rectangular & 0.61 & Low & Medium & $0.529-0.379$ & $0.263-0.151$ \\
\hline Rectangular & 0.61 & Low & Large & $0.529-0.379$ & $0.382-0.309$ \\
\hline Rectangular & 0.61 & Standard & Small & $0.7-0.667$ & $0.051-0.03$ \\
\hline Rectangular & 0.61 & Standard & Medium & $0.7-0.667$ & $0.221-0.171$ \\
\hline Rectangular & 0.61 & Standard & Large & $0.7-0.667$ & $0.407-0.305$ \\
\hline Rectangular & 0.61 & High & Small & $0.798-0.745$ & $0.049-0.032$ \\
\hline Rectangular & 0.61 & High & Medium & $0.798-0.745$ & $0.215-0.167$ \\
\hline Rectangular & 0.61 & High & Large & $0.798-0.745$ & $0.438-0.329$ \\
\hline
\end{tabular}

a gauge with $1 \mathrm{~mm}$ resolution from the gate zero opening identified on the gate stem, to the reference location on the lift nut. Net gate opening area was computed using the gate opening and pipe inside diameter using Eq. (3).

\section{Flow Measurement Errors and Uncertainty}

Volumetric uncertainties are discussed in detail in the companion paper. Similar statistical procedures are used in this evaluation. Coefficient of discharge $\left(C_{d}\right)$ values were computed for each net gate opening under each scenario in Table 1.

The percent error between the computed flow rate and the measured flow rate through the mag meters is computed based as

$$
E_{Q i}=\frac{Q_{i}-Q}{Q} \times 100
$$

where $E_{Q i}=$ percent error between the estimated flow $\left(Q_{i}\right)$ and the actual flow measured by the mag meter $(Q)$. The estimated flow $\left(Q_{i}\right)$ was based on the new $C_{d}$ values developed from this work $\left(Q_{\text {improved }}\right)$. The relative expanded uncertainty (95\% confidence level) was developed based on multiple independent tests with the same gate at each gate opening for the flow rate using the new $C_{d}$ values from this study. Standard uncertainty of the meter gate flow rate $\left(U_{Q}\right)$ was computed as the standard deviation of the error $\left(Q_{i^{-}} Q\right)$ at each gate opening. A coverage factor of $k=2$ (i.e., \pm 2 standard deviations) was applied for the expanded uncertainty to the $95 \%$ confidence level $\left(U_{Q_{-} 95}\right)$. The relative expanded uncertainty $\left(R U_{95}\right)$ was computed as the relative expanded uncertainty $\left(U_{Q_{-} 95}\right)$ divided by the main flow rate for the tests for that gate opening. More discussion on the methods used can be found in the companion paper as well as other references (Taylor and Kuyatt 1994; USBR 1997; Lozano et al. 2009).

\section{Results and Discussion}

The $C_{d}$ values computed from Eq. (2), for each testing scenario, are shown at different fractions of net open areas $\left(A_{o} / A_{p}\right)$ for the 0.46 and $0.61 \mathrm{~m}$ rectangular gates in Figs. 2(a and c), respectively. These $C_{d}$ values are based on a head difference measurement using the downstream water level at the $0.305 \mathrm{~m}$ (12-in.) tap location. The net gate open area $\left(A_{o}\right)$ is computed from Eq. (3) and the pipe inside diameter area was computed using the pipe inside radius $\left(R_{p}\right)$ and the formula for area of a circle.

Figs. 2(a and c) indicate significant variability at the lowest gate opening of $0.05 \mathrm{~m}$ ( $2 \mathrm{in}$.), which for these rectangular gates resulted in an $A_{o} / A_{p}$ of less than 0.10 . There are several reasonable explanations for this variability. Gate leakage through areas other than the pipe open area resulting as a higher percentage of the total flow could be one cause for the variability. This could depend on the head difference $(\Delta H)$ either causing increased or decreased leakage. Both the rectangular and Armco-type (round) gates use a wedge-type seating mechanism that forces the gate to seal when completely closed. When it is opened, however, water can leak between the gate and the seal around the perimeter of the pipe. Summers (1951) also found inconsistent $C_{d}$ values at low gate openings with the Armco-type gates.

Another potential cause is the hydraulics downstream of the gate at the stilling well tap location. The water jets through the bottom of the gate creating a roller structure (where velocities are circulating in a reverse direction) in the water between the jet and the top of the pipe. While this roller structure would occur in most gate openings, it is most pronounced at the smallest openings. This could be the cause of inconsistent downstream head readings as is indicated in $A_{o} / A_{p}$ less than 0.10 in Figs. 2(a and c). Therefore, it is recommended that $A_{o} / A_{p}$ should be greater than 0.10 for rectangular gates, which is equivalent to a net gate opening of $0.10 \mathrm{~m}$ ( $4 \mathrm{in}$.) or more for the $0.46 \mathrm{~m}$ and $0.61 \mathrm{~m}$ gates. The smallest net gate opening was removed from the analysis and the remaining $C_{d}$ values are shown in Figs. 2(b and d).

The $C_{d}$ values in Fig. 2 are higher at smaller $A_{o} / A_{p}$, tend to level out, and then increase again at larger $A_{o} / A_{p}$. For the $0.61 \mathrm{~m}$ gate, the $C_{d}$ values are relatively consistent at $A_{o} / A_{p}$ greater than 0.20 and less than 0.80. Ball (1961) found similar results at the larger gate openings, where the $C_{d}$ values increase and then drop down at full opening $\left(A_{o} / A_{p}=1\right)$. He attributed this variation to the tap location where the downstream water level is measured. This will be discussed in more detail later in this section. The gate structure seems to play a role since the increase in $C_{d}$ at the higher gate openings is not as significant for the Armco-type gates described in the companion paper.

Similar to the statistical analysis in the companion paper, a multiple regression analysis was examined on the non-excluded 

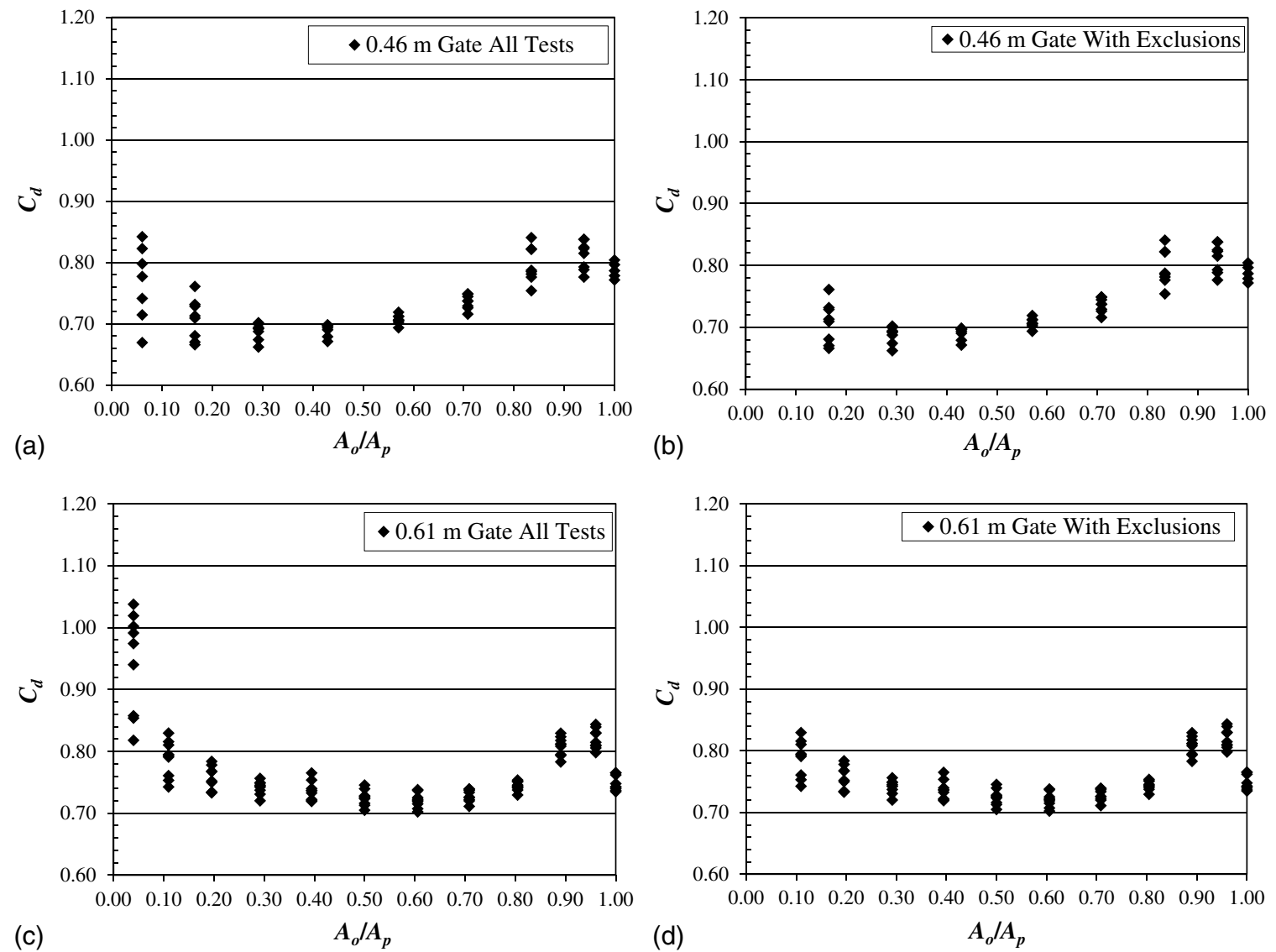

Fig. 2. Coefficient of discharge for all rectangular testing scenarios (a and c) and excluding fractions of net gate openings less than 0.2 and tests with upstream head tests less than 0.5 times the pipe diameter $(\mathrm{b}$ and $\mathrm{d})$ related to the fraction of gate open area $\left(A_{o} / A_{p}\right)$ for the rectangular 0.46 and $0.61 \mathrm{~m}$ gates measuring the downstream water level at the $0.305 \mathrm{~m}$ pressure tap

data relating $A_{o} / A_{p}, H_{1} / D$ and $\Delta H / H_{1}$ to $C_{d}$ using the model

$$
\begin{aligned}
\hat{C}_{d}= & \beta_{5} \frac{A_{o}}{A_{p}}+\beta_{4} \frac{A_{o}}{A_{p}}+\beta_{3} \frac{A_{o}}{A_{p}} \\
& +\beta_{2} \frac{H_{1}}{D}+\beta_{1} \frac{\Delta H}{H_{1}}+\beta_{0}
\end{aligned}
$$

where $\hat{\mathrm{C}}_{d}=$ predicted discharge coefficient; $\beta_{0}$ through $\beta_{5}=$ regression coefficients; and other variables have been previously defined. Residual analysis was used to confirm the assumptions (normality, homoscedasticity, and independence of the errors) required for the multiple regression. The multiple regression coefficients and corresponding $P$-values for each gate size tested are shown in Table 2. It can be concluded that $A_{o} / A_{p}$ have some influence on $C_{d}$, ( $P$-value less than 0.01$)$ while statistically, $H_{1} / D$ and $\Delta H / H_{1}$ do not affect $C_{d}$. Additionally, since the $\beta_{5} P$-value for the $0.61 \mathrm{~m}$ gate is greater than 0.01 , the null hypothesis that $\beta_{5}$ is zero cannot be rejected (at an $\alpha$-level of 0.01 ). The low $R^{2}$ value for the $0.61 \mathrm{~m}$ gate is due to the relatively constant $C_{d}$ between $A_{o} / A_{p}$ of $0.2-0.8$. Multiple regression is not recommended to compute the $C_{d}$. Alternative recommendations for determining $C_{d}$ values will be discussed.

Fig. 3 shows the $C_{d}$ related to the relative upstream head $\left(H_{1} / D\right)$. Different fractions of net opening areas $\left(A_{o} / A_{p}\right)$ are shown for each rectangular gate size. The regression analysis results in Table 2 indicate that $H_{1} / D$ does not affect $C_{d}$ statistically. Additionally, performance does not seem to be impacted for $H_{1} / D$ greater than 0.5 . This indicates that upstream head $\left(H_{1}\right)$ can be lower than the current recommendation of 1 pipe diameter but should remain at or above 0.5 times the pipe diameter. Having an $H_{1}$ that is too low may lead to vortexing in the supply canal (which introduces air), and also limits the $\Delta H$ because the downstream water level becomes too low and the difference also cannot be accurately measured.

Relationships between $C_{d}$ and relative head difference $\left(\Delta H / H_{1}\right)$ and Reynolds number in the turnout pipe $\left(\mathrm{R}_{\text {pipe }}\right)$ are shown in Figs. 4(a-d). The $C_{d}$ at the $0.305 \mathrm{~m}$ tap location is not affected by relative head difference at an $\alpha$-level of 0.1 (Table 2).

Table 2. Results of a Multiple Regression Analysis Showing the Test Variables Influencing $C_{d}$ Values for Each Rectangular Gate Tested. $P$-Values $>0.01$ Indicate the Variable does not Affect $C_{d}$ at an

\begin{tabular}{|c|c|c|c|c|c|}
\hline \multirow[b]{2}{*}{ Predictor } & \multirow[b]{2}{*}{ Coefficient } & \multicolumn{2}{|c|}{$0.46 \mathrm{~m}$ rectangular ${ }^{\mathrm{a}}$} & \multicolumn{2}{|c|}{$0.61 \mathrm{~m}$ rectangular ${ }^{\mathrm{b}}$} \\
\hline & & Coefficient & $P$-value & Coefficient & $P$-value \\
\hline Constant & $\beta_{0}$ & 0.815 & 0.000 & 0.868 & 0.000 \\
\hline$\left(A_{o} / A_{p}\right)^{3}$ & $\beta_{5}$ & -0.823 & 0.000 & -0.373 & 0.020 \\
\hline$\left(A_{o} / A_{p}\right)^{2}$ & $\beta_{4}$ & 1.673 & 0.000 & 0.936 & 0.001 \\
\hline$\left(A_{o} / A_{p}\right)$ & $\beta_{3}$ & -0.859 & 0.000 & -0.622 & 0.000 \\
\hline$H_{1} / D$ & $\beta_{2}$ & 0.005 & 0.553 & -0.006 & 0.652 \\
\hline$\Delta H / H_{1}$ & $\beta_{1}$ & -0.023 & 0.390 & -0.037 & 0.018 \\
\hline
\end{tabular}
$\alpha$-Level $=0.01$ 

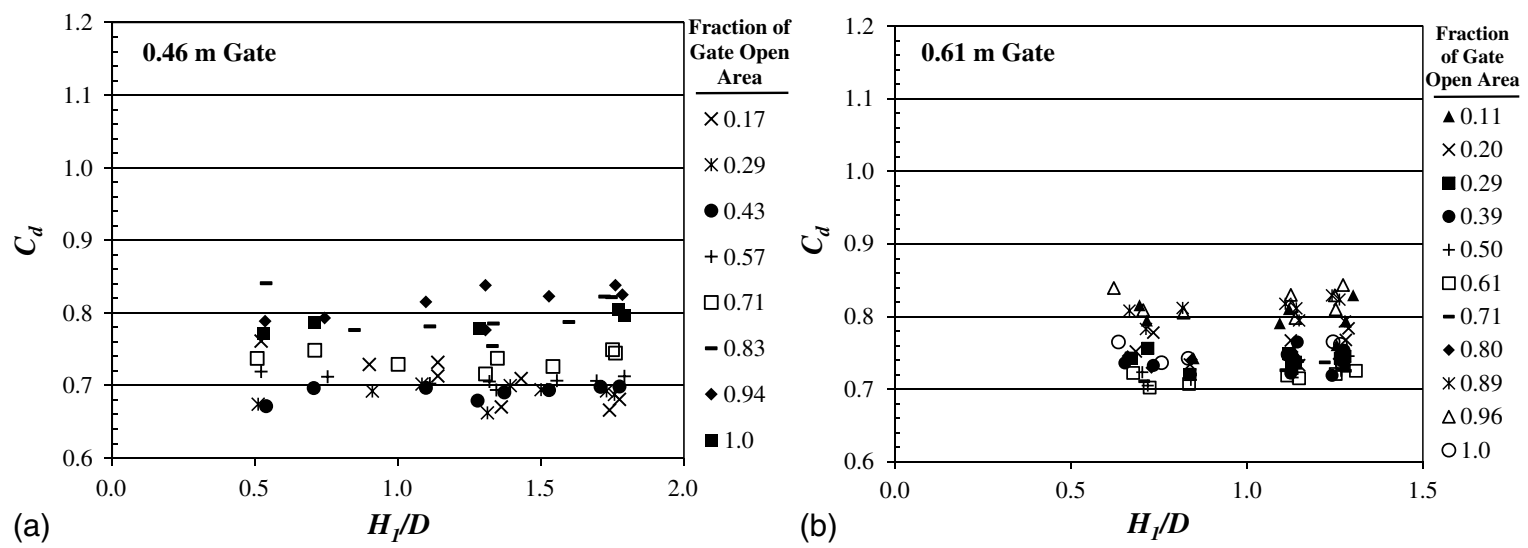

Fig. 3. Coefficient of discharge variation ( $0.305 \mathrm{~m}$ pressure tap location) at different relative upstream head $\left(H_{1} / D\right)$ for the fraction of gate openings greater than 0.10: (a) rectangular $0.46 \mathrm{~m}$ gate; (b) rectangular $0.61 \mathrm{~m}$ gate
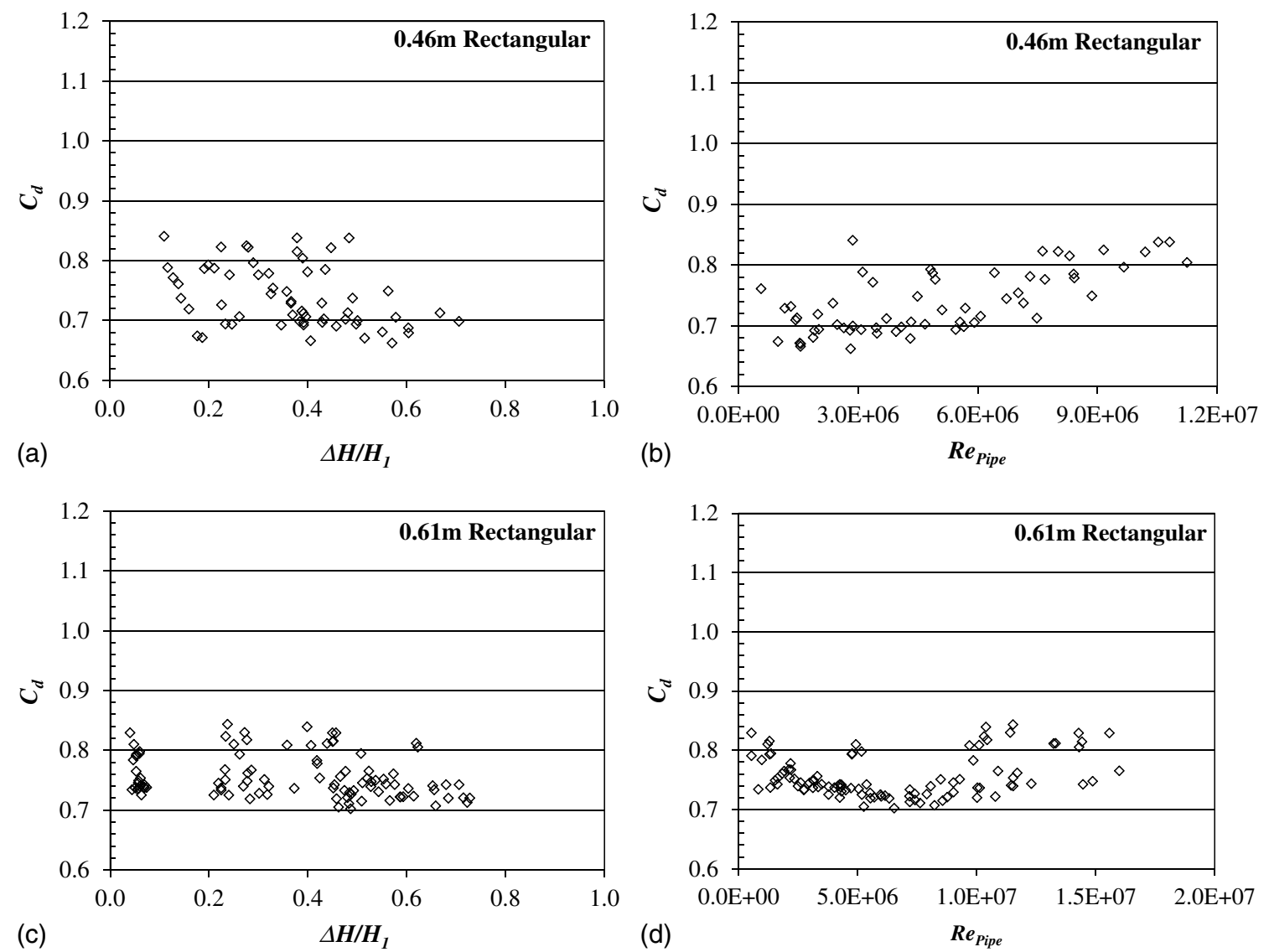

Fig. 4. Coefficient of discharge relationship to relative head difference $\left(\Delta H / H_{1}\right)$ and Reynolds number in the turnout pipe $\left(\mathrm{R}_{\text {pipe }}\right)$ for the two rectangular gate sizes

The $P$-value was low for the $0.61 \mathrm{~m}$ rectangular gate $\beta_{1}$ (but still above the selected $\alpha$-level of 0.01). While this could be interpreted as being worthy of further investigation, the small coefficient $\left(\beta_{1}\right)$ indicates that any potential effect $\Delta H / H_{1}$ has on $C_{d}$ is very small. As discussed in the companion paper, $\Delta H / H_{1}$ above 0.75 was attempted but the water level was too low in the stilling well attached to the $0.305 \mathrm{~m}$ tap to make an accurate reading. In application, care should be taken to ensure that the upstream head above the pipe $\left(H_{1}\right)$ is high enough so that the downstream water level can be measured.
Figs. 4(b and d) indicate that $C_{d}$ increases at higher $\mathrm{R}_{\text {pipe }}$. This is likely driven by gate opening (Fig. 2) since higher $R_{\text {pipe }}$ coincides with the larger gate openings in general. Because of this correlation $R_{\text {pipe }}$ was not included in the multiple regression analysis.

The average $C_{d}$ values based on a $0.305 \mathrm{~m}$ (12-in.) downstream tap location by $A_{o} / A_{p}$ for the two rectangular gate sizes are shown in Fig. 5(a). At $A_{o} / A_{p}$ less than 0.6, the larger $0.61 \mathrm{~m}$ (24-in.) gate $C_{d}$ is higher than the $0.46 \mathrm{~m}$ rectangular gate. The $C_{d}$ values are similar at $A_{o} / A_{p}$ greater than 0.6 and less than 1.0. These results 

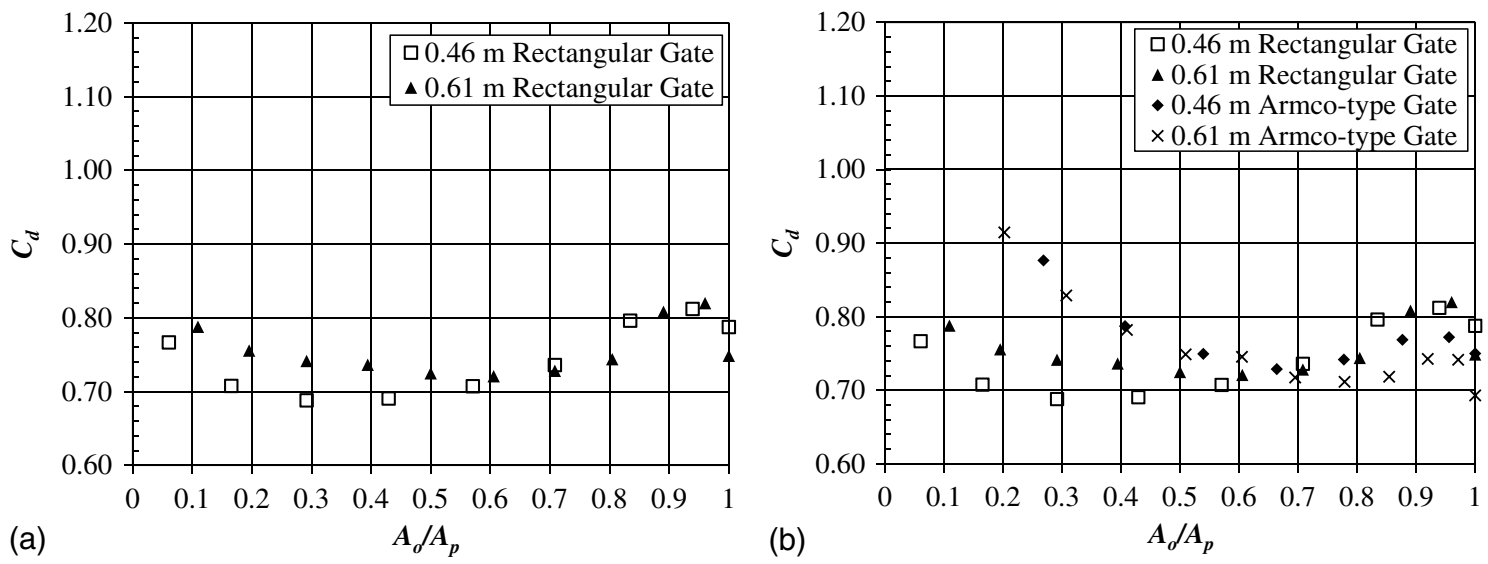

Fig. 5. Average $C_{d}$ computed in this study for the rectangular gates tested (a) and rectangular compared with Armco-type (round) gates of the same size from a companion paper (b) by fraction of net gate opening area

differ from those found for the Armco-type (round) gate presented in the companion paper as shown in Fig. 5(b). The Armco-type $C_{d}$ values are similar between the two gate sizes for $A_{o} / A_{p}$ less than 0.7 and deviate at the larger gate opening areas.

It is clear from Fig. 5(b) that the gate shape plays a role in $C_{d}$. This is likely due to the influence of the bottom of the gate on the water entering the pipeline related to the location of the stilling well tap. A higher $C_{d}$ value for the Armco-type gates at $A_{o} / A_{p}$ less than 0.7 indicates that there is less head difference measured at the $0.305 \mathrm{~m}$ (12-in.) pressure tap compared with the rectangular gates for the same flow rate.

The difference in $C_{d}$ values when the gate is fully open (bottom of the gate is at the top of the pipe) where $A_{o} / A_{p}$ is equal to 1 . The $C_{d}$ values should be similar for the same size pipes; however, the Armco-type gates have $C_{d}$ values lower than their rectangular counterparts. Again this indicates that gate shape, even though the gate is not protruding into the pipe area, is influencing the water movement into the pipe. A more detailed examination of tap location influence on $C_{d}$ with different gate sizes and types will be conducted in future work.

The uncertainty evaluation was conducted using the $C_{d}$ values developed in this study [Fig. 5(a)] to compute the flow rate from the testing scenarios compared with the flow rate measured through the mag meters. Since the $C_{d}$ values were developed using the same data, the average percent error is zero as shown in Figs. 6(a and b). Of importance is the uncertainty due to the variability in $C_{d}$ values under various $A_{o} / A_{p}$. As indicated in Fig. 2, the larger spread in $C_{d}$ values at the lower and higher gate openings results in greater relative expanded uncertainty under these conditions. However, with $A_{o} / A_{p}$ greater than 0.1 (equivalent to gate openings of $4 \mathrm{in}$. or greater for rectangular gates), the relative expanded uncertainty is less than $\pm 10 \%$. For the majority of $A_{o} / A_{p}$, the expanded relative uncertainty is within $\pm 5 \%$.

A comparison of the flow rate computed from USBR Water Measurement Manual (Chapter 9. Section 14. Meter Gates) $C_{d}$ values based on full pipe area and on the pressure tap location of $D / 3$ (where $D$ is the pipe diameter) was conducted and is shown in Figs. 6(c and d). The USBR $C_{d}$ method performed relatively well for the $0.46 \mathrm{~m}$ (18-in.) rectangular gate at openings less than $0.41 \mathrm{~m}$ (16 in.) with a slight underestimation of discharge (average percent flow rate error was $-3 \%$ ). However, the USBR $C_{d}$ showed significant underestimation of flow rates through the meter gate with an average percent flow rate error of $-10 \%$ with gate openings less than $0.508 \mathrm{~m}$ (20-in. opening).

\section{Downstream Pressure Tap Location Influence}

While a more in-depth evaluation of pressure tap locations is planned in the future using data collected in this study, existing meter gates may have tap locations other than $0.305 \mathrm{~m}$. It is the author's experience that the tap location is often less than the recommended $0.305 \mathrm{~m}$. Fig. 7 shows the variation in $C_{d}$ for different tap locations for the two rectangular gates. The relationship between tap location and $C_{d}$ was similar for the equivalent Armco-type (round) gates shown in the companion paper. The $0.46 \mathrm{~m}$ (18-in.) gate shows close agreement for $C_{d}$ values at lower gate openings $\left(A_{o} / A_{p}\right.$ less than $0.5)$. At larger openings the $C_{d}$ values tend to deviate. However, the $0.20 \mathrm{~m}$ (8-in.) tap location has similar $C_{d}$ values to the $0.305 \mathrm{~m}$ location at $A_{o} / A_{p}$ less than 0.8 . In contrast, as with the similar sized Armco-type gate, the $0.61 \mathrm{~m}$ (24-in.) gate indicated little influence between tap location and $C_{d}$ at $A_{o} / A_{p}$ less than 0.75 .

At higher gate openings, the influence of tap location on $C_{d}$ is evident. This indicates that the water jetting under the bottom of the gate, and the jet connecting back up to the pipe downstream, is influencing the downstream water level at different locations. The point where this jet connects with the top of the pipe depends on gate opening. At smaller gate openings the water would connect further downstream than at larger gate openings.

The recommendation by Ball (1961) that the preferred tap location be a distance of $D / 3$ cannot be validated in this work. The $D / 3$ location is equivalent to the 0.15 and $0.20 \mathrm{~m}$ tap locations for the 0.46 and $0.61 \mathrm{~m}$ gate, respectively. While there is something to be said about the behavior of the $C_{d}$ at the closer tap location, it can be difficult in field applications to access the pipe a close distance from the back face of the gate due to the thickness of the concrete bulkhead commonly used.

If a $0.46 \mathrm{~m}$ (18-in.) rectangular gate is used with a stilling well location less than $0.20 \mathrm{~m}$ ( 8 in.), the tap location should be moved closer to the $0.305 \mathrm{~m}$ (12-in.) location. This will help ensure accurate discharge is recorded using the $C_{d}$ values presented in this study that are based on the $0.305 \mathrm{~m}$ tap location. For both gate sizes with tap locations less than $0.305 \mathrm{~m}$ downstream from the back face of the gate, $A_{o} / A_{p}$ should be limited to less than 0.80 (less than 14-in. opening for the 18-in. gate and 18-in. opening for the 24-in. gate).

\section{Application}

The companion paper provides a set of detailed guidelines for the use of meter gates to attain accurate discharge measurements. 

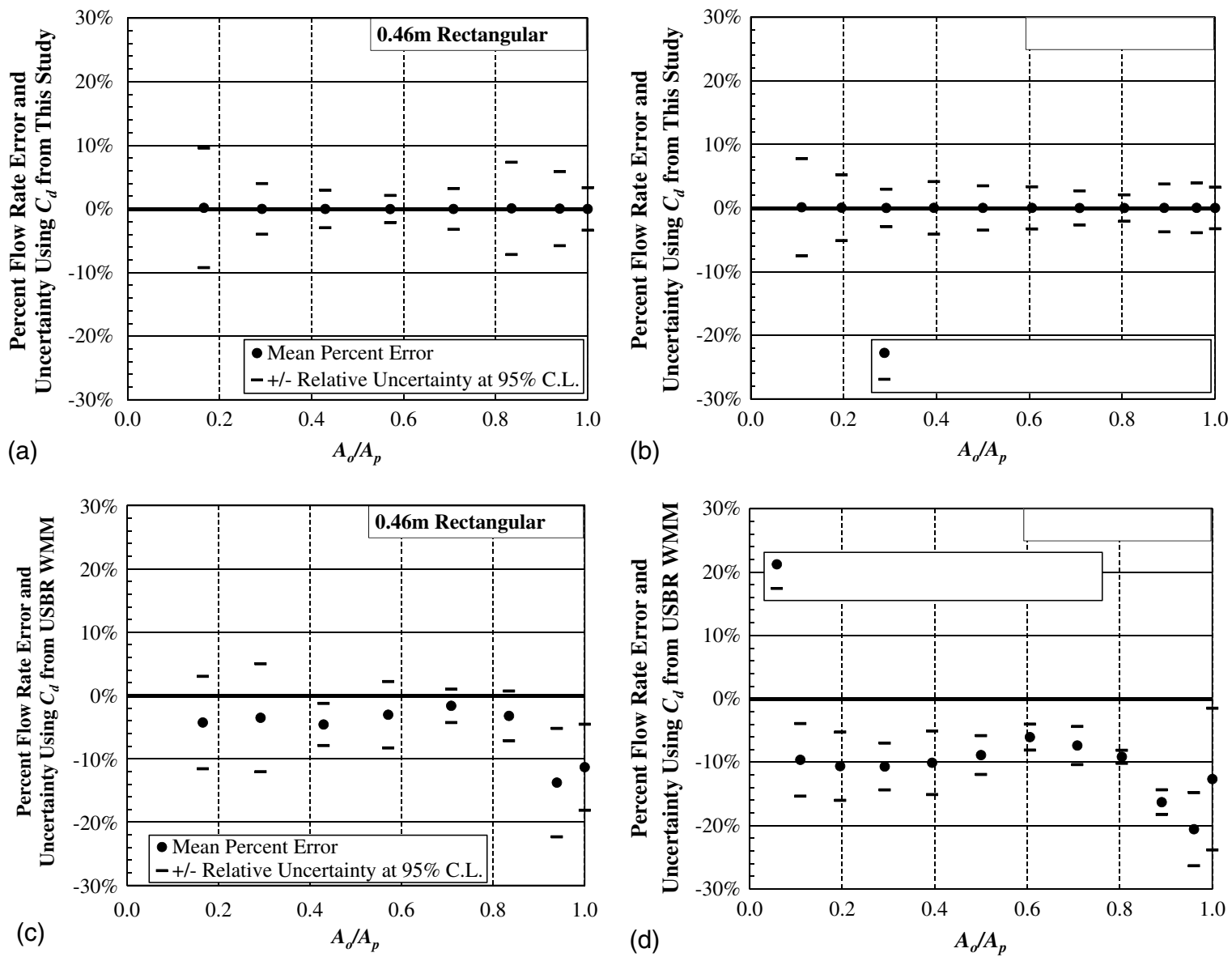

Fig. 6. Average relative error for the two rectangular gates tested based on the new $C_{d}$ values from this study; relative expanded uncertainty (95\% confidence level) is shown as error bars to indicate the accuracy of the instantaneous flow measurement at different gate opening areas
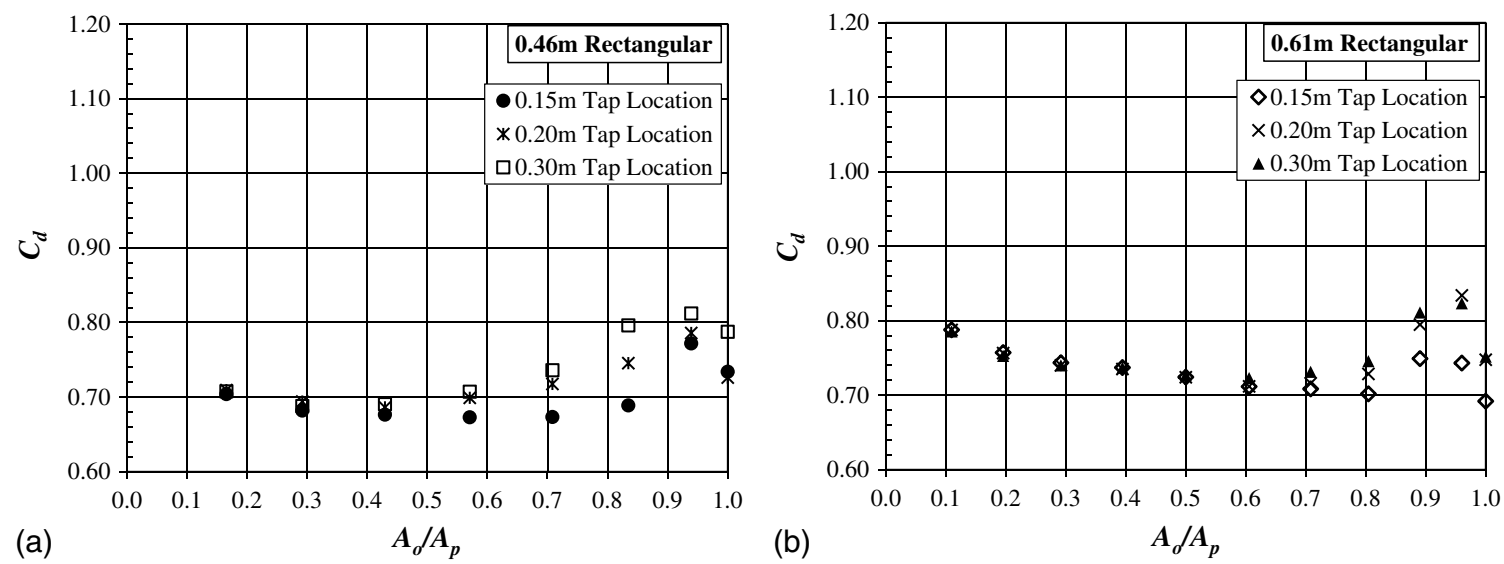

Fig. 7. Effects of downstream tap location on $C_{d}$ for the two rectangular gates at different net gate opening fractions

That section will not be repeated since those recommendations and limitations are the same for the rectangular gates presented here and the Armco-type (round) gates presented in the companion paper. Proper identification of the zero gate opening is critical for obtaining accurate discharge measurement. If the guidelines in the companion paper are followed, users of rectangular gate discharge rating tables based on the $C_{d}$ values shown in Table 3 should expected uncertainties less than $\pm 10 \%$ at all $A_{o} / A_{p}$ greater than 0.10 and better than $\pm 5 \%$ at more at $A_{o} / A_{p}$ greater than 0.20 and less than 0.80 . The relationship between $A_{o} / A_{p}$ and actual gate opening in meters and inches is shown in Table 3. It is reasonable to assume that most gates would be opened to a variety of gate openings over the course of a season, so an average uncertainty over all gate openings of $\pm 5 \%$ can be expected. However, this should be examined on a case-by-case basis.

Table 3 shows the new $C_{d}$ values for the two rectangular gates in this study by gate opening, fraction of net gate opening $\left(y / y_{p}\right.$, where $y_{p}$ is the pipe inside diameter) and $A_{o} / A_{p}$. It is 
Table 3. New $C_{d}$ Values from This Study by Net Gate Opening $(y)$, Fraction of Net Gate Opening $\left(y / y_{p}\right)$, and Fraction of Net Opening Area $\left(A_{o} / A_{p}\right)$

\begin{tabular}{lccccc}
\hline Gate size & $y(\mathrm{~m})$ & $y$ (in.) & $y / y_{p}$ & $A_{o} / A_{p}$ & $C_{d}$ \\
\hline $0.46 \mathrm{~m}$ (18-in.) & 0.102 & 4 & 0.222 & 0.165 & 0.708 \\
& 0.152 & 6 & 0.333 & 0.292 & 0.688 \\
& 0.203 & 8 & 0.444 & 0.429 & 0.690 \\
& 0.254 & 10 & 0.556 & 0.571 & 0.707 \\
& 0.305 & 12 & 0.667 & 0.708 & 0.736 \\
& 0.356 & 14 & 0.778 & 0.835 & 0.796 \\
& 0.406 & 16 & 0.889 & 0.939 & 0.812 \\
& 0.457 & 18 & 1.000 & 1.000 & 0.788 \\
& 0.102 & 4 & 0.167 & 0.110 & 0.788 \\
& 0.152 & 6 & 0.250 & 0.196 & 0.756 \\
& 0.203 & 8 & 0.333 & 0.292 & 0.741 \\
& 0.254 & 10 & 0.417 & 0.394 & 0.736 \\
& 0.305 & 12 & 0.500 & 0.500 & 0.725 \\
& 0.356 & 14 & 0.583 & 0.606 & 0.721 \\
& 0.406 & 16 & 0.667 & 0.708 & 0.728 \\
& 0.457 & 18 & 0.750 & 0.804 & 0.744 \\
& 0.508 & 20 & 0.833 & 0.890 & 0.808 \\
& 0.559 & 22 & 0.917 & 0.960 & 0.820 \\
& 0.610 & 24 & 1.000 & 1.000 & 0.748 \\
\hline
\end{tabular}

recommended that these be used for creating new rating tables for these gates. While a best-fit polynomial can be created for each gate, it is more appropriate to interpolate between these values to estimate $C_{d}$ values for other gate openings. A best-fit regression may have a high $R^{2}$ value but the $C_{d}$ values will not match between the regression equation and the tabular values below at all gate openings, leading to increased uncertainty. In addition, it can perform very poorly at gate openings less than $0.05 \mathrm{~m}$. Linear interpolation or a more advanced interpolation method can be used. If an advanced interpolation is used, the values should be plotted with those reported in this table to ensure that the results conform.

\section{Conclusion}

In this study, new coefficient of discharge values based on actual net gate open area for $0.46 \mathrm{~m}$ (18-in.) and $0.61 \mathrm{~m}$ (24-in.) rectangular meter gates were developed based on a $0.305 \mathrm{~m}$ (12-in.) downstream pressure tap location. The gates were tested under a variety of conditions at different gate openings. The $C_{d}$ values varied most significantly at the lowest net gate opening, increasing the uncertainty at $A_{o} / A_{p}$ less than 0.20 . Higher gate openings for the $0.46 \mathrm{~m}$ gate also showed increased variability; however, the increase in uncertainty was still within $\pm 7 \%$ or better. Average overall uncertainty (average of all gate openings) was within $\pm 5 \%$.

The $C_{d}$ values differed significantly between the $0.46 \mathrm{~m}$ and the $0.61 \mathrm{~m}$ rectangular gates for most openings. This is in contrast to what Ball (1961) found in earlier work. Even though Ball recommended different downstream pressure tap locations, Fig. 7 indicates a significant departure between the $C_{d}$ values for the different gate sizes. The USBR Water Measurement Manual meter gate $C_{d}$ figure should not be used for estimating $C_{d}$ values for rectangular meter gates of various sizes. The authors recommend using the $C_{d}$ values from this study for $0.46 \mathrm{~m}$ and $0.61 \mathrm{~m}$ rectangular gates to compute the flow rates if using these as meter gates.

Based on other data collected in this study, an evaluation of the impacts on supply channel velocity perpendicular to the gate will be conducted. Future work is needed to evaluate the effects of different entrance conditions on gate discharge ratings with the supply channel perpendicular to the meter gate turnout. In addition, further testing of different gate sizes is needed to either determine the appropriate discharge rating or examine if a relationship between gate size and coefficient of discharge can be developed.

\section{Acknowledgments}

This work was funded by a grant (13-01-005) from the California State University Agricultural Research Institute (CSU ARI). The authors would also like to acknowledge the support provided by San Luis Canal Company, including the donation of the rectangular gates. This work could not have been completed without the hours of work provided by Cal Poly water engineering and irrigation graduate students and construction efforts by ITRC student employees from the BioResource and Agricultural Engineering Department.

\section{Notation}

The following symbols are used in this paper:

$A_{o}=$ net gate opening area;

$A_{p}=$ full pipe area;

$C_{d}=$ coefficient of discharge;

$C_{v}=$ coefficient of velocity;

$D=$ pipe diameter;

$E_{Q i}=$ percent error between the estimated flow and the actual flow;

$g=$ gravitational acceleration;

$H_{1}=$ upstream head;

$\Delta H=$ head difference across the gate;

$Q=$ flow rate;

$Q_{i}=$ estimated flow rate;

$Q_{\text {improved }}=$ flow rate estimated from new $C_{d}$ values developed from this work;

$\mathrm{R}_{\text {pipe }}=$ Reynolds number in the turnout pipe;

$R_{p}=$ inside radius of the pipe;

$R U_{95}=$ relative expanded uncertainty;

$U=$ uncertainty;

$U_{Q}=$ instantaneous flow measurement accuracy;

$U_{Q \_95}=$ expanded uncertainty to the $95 \%$ confidence level; and $y=$ net gate opening.

\section{References}

Ball, J. W. (1961). "Flow characteristics and limitations of screw lift vertical metergates." Rep. No. HYD-471, Bureau of Reclamation, Denver, 53.

Lozano, D., Mateos, L., Merkley, G. P., and Clemmens, A. J. (2009). "Field calibration of submerged sluice gates in irrigation canals." J. Irrig. Drain. Eng., 10.1061/(ASCE)IR.1943-4774.0000085, 763-772.

Skogerboe, G. V., and Merkley, G. P. (1996). "Irrigation maintenance and operations learning process." Water Resources Publication, LLC, Highlands Ranch, CO.

Summers, J. B. (1951). "Flow characteristics and limitations of Armco meter gates; August 5, 1951.” Rep. No. HYD-314, Bureau of Reclamation, Denver.

Taylor, B. N., and Kuyatt, C. E. (1994). "Guidelines for evaluating and expressing the uncertainty of NIST measurement results." NIST Technical Note 1297, NIST, Gaithersburg, MD.

USBR. (1997). "Water measurement manual: A guide to effective water measurement practices for better water management." A Water Resources Technical Publication, U.S. Dept. of the Interior, Bureau of Reclamation, Denver. 